\title{
David M. Dines, Cato T. Laurencin, Gerald R. Williams (eds): Expert consult arthritis and arthroplasty: the shoulder
}

\author{
Imprint: Saunders Elsevier, Philadelphia, Hardcover, 336 pages, publication date: \\ JUL-2009 + DVD, 180,41 €, ISBN-13: 978-1-4160-4975-3, ISBN-10: 1-4160-4975-4
}

Pierre Kehr

Received: 1 May 2010/Accepted: 5 May 2010/Published online: 19 May 2010

(C) Springer-Verlag 2010

The authors David Mr. Dines, Cato. T. Laurencin, and Gerald R. Wiliams present in their book the whole of the knowledge validated to date on a subject extremely interesting and difficult namely: omarthrosis and arthroplasties. The book is at the same time exhaustive and digest with a strong practical accent.

Each chapter is written by personalities recognized like having largest experience under respective fields.

$\mathrm{He}$ is recalled to the beginning of the book briefly the place of the preserving treatment and nonprosthetic but the sharp one of the subject is quickly reached. The principles of the total arthroplasty whose bases were established by Neer are developed thoroughly with an accent on the analysis of the glenoid cavity, the demystification of the primary glenoid grafting, thus making it possible to increase prosthetic longevity and to decrease the risks of instability.

The less invasive techniques and which preserve the osseous capital, like the resurfacing of the humeral head, became now a great classic thanks to Stephen Copeland. In the same spirit, the techniques of biological resurfacing of the glenoid cavity are discussed more and more among young patients but much of controversies still remain.

An important chapter has as a subject the complications and the revisions of the arthroplasties of the shoulder. It reflects current reality, good number of prostheses installation these last years requiring of the revisions. There too progress is obvious. The revision by reversed arthroplasty associated with a rebuilding of the glenoid cavity with grafting falls under this register.

Apart from the quality of the rigor and exhaustiveness of the subjects presented, one notices a pleasant form, an abundance of the illustrations allowing thus easily to include/understand a so complex topic. In a certain manner, this work constitutes a true bible.

\section{Dr. Christian Cuny}

Metz

No funds were received in support of this study.
P. Kehr ( $\square)$

SOTEST, Strasbourg, France

e-mail: kehrpier@aol.com 